\title{
»Eene meene muh - drin bist Du « Zur Wiederkehr der Sicherungsstrafe in der gegenwärtigen Diskussion
}

In seiner Kritik an der in den USA wieder hoffähigen Ideologie des Unschädlichmachens mehrfach rückfälliger Täter, "selective incapacitation «, zeigt Karl Schumann* die empirische Fragwürdigkeit der üblichen Gefährlichkeitsprognosen. Ihre Fehlerquoten seien so hoch, daß es unverantwortlich sei, eine so schwerwiegende Freiheitsentziehung auf sie zu stützen. Zwar spiele Sicherung als Strafzweck in Deutschland zur Zeit noch keine dominante Rolle, aber dies könne sich ändern. Für Anhänger einer rein zweckmäßigen Prävention ist sie aber unterschwellig in ihrer Bedeutung nicht zu unterschätzen. Daher soll Schumanns Kritik aufgegriffen und straftheoretisch vertieft werden.

\section{Prävention und Täterorientierung:}

Strafzumessungsstudien ergeben für Deutschland, daß etwa nach jeder dritten einschlägigen Vorverurteilung das Risiko steigt, als "unverbesserlich “ ${ }^{1}$ eingestuft und entsprechend härter angepackt zu werden; und zwar unabhängig davon, ob das jeweilige Gericht eher hart oder milde, eher einer generalpräventiven oder einer spezialpräventiven Straftheorie folgt. Die zugrunde gelegte Straftheorie scheint irrelevant oder zumindest nachrangig zu $\operatorname{sein}^{2}$. Dieser nivellierende Effekt einschlägiger Vorstrafen sollte zu denken geben. Er relativiert nämlich die optimistischen Erwartungen, die Reformer vor über zwanzig Jahren hatten. Wir erinnern uns an die Stichworte dieser kurzen Phase eines milden Strafklimas (etwa 1968-1975): das Schuldprinzip geriet in Mißkredit, man sprach vom "Siegeszug« des Gedankens der Spezialprävention und hoffte auf einen rationalen Rückzug strafrechtlicher Intervention auf "unerläßlichen Rechtsgüterschutz«. Auch heute gibt es noch Anklänge an diese Tradition, etwa in Formulierungen wie: Schuldprinzip und Generalprävention appellierten an "unzivile Motive wie Rache und Vergeltung « ${ }^{3}$. Aber heute sind es konfliktschlichtende Utopien, man hofft auf restitutive Reaktionsformen wie Täter-

\footnotetext{
KJ $1 / 95$, S. $88 \mathrm{ff}$.

I Der Ausdruck stammt aus der zu Unrecht als liberale Errungenschaft gefeierten Praventionstheorie Franz von Liszts (Marburger Programm, ZStW 1882, iff.). Die Doktrin der Zweckstrafe ist nicht ablosbar von Liszts Tatertypologie: Besserung der besserungsfahigen und besserungsbedurftigen/Abschrechung der nıcht besserungsbedurftigen / Unschadlichmachung der nicht besserungsfahigen Verbrecher . Dreı Vorverurteilungen waren fur von Liszt dic magische Grenze. Seine Schatzungen der Zahl der zu sichernden sind grauenerregend: die off $1 z ı$ ellen Daten der Rechtspflegestatistık beweısen, meinte er unkritisch, daß "mındestens die Halfte jener Personen, welche Jahr aus, Jahr ein unsere Strafanstalten bevolkern, unverbesserliche Gewohnheitsverbrecher sind. « Vgl. zum historischen Hintergrund der Zweckstrafe, des Schulenstreits und der spateren Rezeption Frommel, wie Fn. 8.

2 Die sog. Irrelevanzthese (oder unfreundlicher ausgedruickt: der Schulenstrett dient weniger der Klarung eines Problems, als vielmehr der Selbstinszenterung und Reputation - Schulenbildung - seiner Protagonisten) wurde schon zu Beginn des Schulenstreits (etwa 1902) von dem als Freirechter nur verkurzt wahrgenommenen H. U. Kantorowicz vertreten, vgl. dens., Monatsschrift fur Kriminologie und Strafrechtsreform 4, 1907/08, S. $68 \mathrm{ff} ; 7,1911$, S. $257 \mathrm{ff}$.

Die Konvergenz der Straftheorien bei der Strafzumessung wurde spater - an entlegener Stelle - empirisch bestatigt: Opp/Peuckert, Ideologie und Fakten in der Rechtsprechung, I971. Mittlerwelle kann die Irrelevanzthese als erwiesen gelten, vgl, den umfassenden Berıcht uber den Stand der Forschung und die Ergebnisse eigener Studien von H.-J. Albrecht, Strafzumessung bei schwerer Krimınalitat, 1994 .

3 So Gunter Frankenberg, Die Zivilgesellschaft in Bedrangnis, Die ZEIT Nr. 47 v. 18.11. 1994, S. 60.
} 
Opfer-Ausgleich u.ä., während es in den 1970er Jahren der rationalisierende Anspruch utilitaristischer Strafbegründungen war, der den geläuterten, "modernen" Erwartungen entgegen zu kommen schien. Sichten wir die mittlerweile unübersichtliche Landschaft. Es sieht so aus, als könnten wir nach hundert Jahren "Schulenstreit « mit beiden Positionen nicht mehr viel anfangen. Wir beginnen zu begreifen, was Émile Durkheim I 893 gemeint haben könnte, als er die These von der "Normalität« der Kriminalität in modernen Gesellschaften aufstellte und meinte, daß nicht nur schwere Formen von Normabweichung notwendig zu jeder Gesellschaft gehören, sondern auch die jeweilige repressive Gegenreaktion. Erst durch sie könne das verletzte Kollektivgefühl (so die Formulierung von Durkheim) wieder hergestellt werden ${ }^{4}$. Lange Zeit erinnerte dieser Satz an Hegels Wiedervergeltungslehre und fiel unter Ideologie- und Idealismusverdacht. Aber wenn man sich von der Vorstellung löst, relative Straftheorien seien "besser" als solche, die im »absoluten « Gewande einhergehen, gewinnt die These von der (kultur- und zeitübergreifenden) Unvermeidlichkeit einer Gegenreaktion, nicht um »Zwecke« zu erreichen, sondern um den normativen Anspruch zu wahren, an Plausibilität.

\section{Die Rolle von Vorstrafen bei der Strafzumessung}

Die empirisch belegte nivellierende Wirkung einschlägiger Vorstrafen bei der Strafzumessung bestätigt die funktionalistische Deutung von Kriminalität und Strafe. Sie zeigt, daß die Strafverfolgung - jenseits aller Absichtserklärungen - einer ungeschriebenen Regel folgt. Sie reagiert auf den wiederholten Normbruch. Nicht die Theorie, sondern das sich wandelnde Strafklima prägt die Intensität und Härte der jeweiligen Anklagepraxis und Strafzumessung. Aber auf den Indikator "Vorstrafenbelastung " reagieren alle Systeme. Ein kurzer Abgleich der zu erwartenden - ritualisierten Strafzumessungsbegründungen mit Blick auf die implizit angewandten Straftheorien bestätigt dies: eine in erster Linie an Resozialisierung orientierte Spezialprävention sieht in Vorstrafen einen schwer widerlegbaren Indikator für eine negative Prognose (mit allen Konsequenzen für die Zuschreibungen auf jeder Stufe der Sanktionenentscheidung: beginnend mit der Frage der Diversion oder förmlichen Reaktion, der Wahl der Strafart und der Strafhöhe). Wer generalpräventiv argumentiert, wird formelhaft behaupten, daß die zurechenbare Normverletzung so schwer wiege, daß eine Einstellung unangemessen und die ausgesprochene Strafe etwa in dieser Höhe erforderlich sei. Wer hingegen liberal argumentiert und vor allem das objektivierbare Kriterium der Deliktschwere betont (dies ist z.Zt. bei den Massedelikten wie Diebstahl u.ä. die dominante Theorie der Praxis, während Tätertypologien eher ihr Unwesen bei den ideologisch besetzten Deliktsbereichen treiben), sieht in der wiederholten Verletzung desselben oder ähnlicher Rechtsgüter einen triftigen Grund für eine belastendere Zurechnung. Die von allen benutzte Formel lautet: der Täter habe sich die Vorverurteilungen nicht zur Warnung dienen lassen.

Liest man diese Floskeln hintereinander, erkennt man, daß sie im Ergebnis auf das-

${ }_{4}$ Grundlegend fur moderne Theorien der indirekten Generalprävention ist nicht der Jurist Jakobs (so aber Schumann, Fn. 7), sondern viel fruher schon der Soziologie É. Durkheim. Alle funktionalistischen Kriminalitats- und Kriminalisierungstheorien implizieren Varianten einer indirekt generalpräventiven Straftheorie, da sie annehmen, daß die Funktion einer Strafe nicht Abschreckung oder Besserung/Sicherung, sondern Normstabilisierung und damit auch ein Versuch der Legitimierung von fundamentalen Normen ist. So explizit Durkheim, de la division du travail social, 1893 (Strafe wird dort verstanden als Ausdruck einer ersten Stufe der Vergesellschaftung, der "mechanischen Solidaritat " einer Gesellschaft); ders. áußerte sich 1895 zur "Normalität« und damit auch zur Notwendigkeit von formalisierten Gegenreaktionen in Form einer rechtlichen Sanktion: Kriminalität als normales Phänomen, dt. übersetzt und abgedruckt in: König (Hrsg.), Soziologische Texte, 1961, S. 156 ff., und in: Sack (Hrsg.), Kriminalsoziologie, 1968, S. 3 ff. Zum historischen Kontext vgl. René König, Émile Durkheim zur Diskussion, 1978. 
selbe hinauslaufen: höhere Strafen bei mehrfach Auffälligen. Im historischen Rückblick sieht man, daß die heute üblichen Begründungsformeln trotz aller Reformen in etwa dem entsprechen, was Anhänger einer - heute nicht mehr vertretenen und auch nicht mehr vertretbaren - "Lebensführungsschuld « in den autoritären bis faschistischen 3 oer und 4 oer Jahren gefordert haben'. Der Unterschied ist der: früher forderte man explizit, was heute implizit - also ohne offen ausgesprochen zu werden - tatsächlich praktiziert wird. Vorstrafen können also entweder mittelbar oder unmittelbar als schulderböhend oder als Indikator für "Gefäbrlichkeit" herangezogen werden.

Eine eigenständige Betonung des Sicherungsgedankens ist also wegen der äquivalenten Funktion des Schuldgedankens und wegen des Leerformelhaften der Spezialprävention für die Praxis der Strafzumessung dogmatisch und sprachlich überflüssig. Hundert Jahre »Schuldstrafrecht « und hundert Jahre »Siegeszug « der Spezialprävention haben beide Begriffe zu Worthülsen und die dahinter stehenden Prinzipien fast inhaltsleer gemacht. Sie können daher Sicherungsaspekte mühelos transportieren. Daher ist die von Schumann angeregte Debatte wichtig. Es ist nämlich nicht auszuschließen, daß sich die Zeiten erneut wandeln und der Gedanke der "Sicherung" auch explizit an Bedeutung gewinnt. Seit 1989 ist das Strafklima - zumindest für sog. nichtdeutsche Tatverdächtige - deutlich härter, Innere-Sicherheits-Debatten sind wieder selbstverständlich geworden. Daher könnten auch historisch überholte Maßnahmen wie die Sicherungsverwahrung wieder gebräuchlicher und das Strafprinzip der "Sicherung der Allgemeinheit « wieder zur rhetorischen Normalausstattung werden. Noch gibt es keine erkennbaren Anzeichen für einen derartigen Paradigmenwechsel, aber es ist dennoch wichtig, grundsätzlich zu überlegen, welche Bedeutung dem Sicherungsaspekt in einem liberal-rechtsstaatlichen Strafrecht zukommen darf.

\section{Wieso ist der Sicherungsgedanke bistorisch Ausdruck illiberaler Tendenzen?}

Aber wie verhält sich diese Befürchtung mit der oben vertretenen Irrelevanzthese? Meine These lautet: "Sicherung « steht historisch für eine angestrebte Tendenzwende in Richtung eines härteren Strafklimas. Die explizite Täterorientierung steht für eine illiberale Haltung. Die Begründung für diese Annahme ist die historische Erfahrung in verschiedenen Rechtskulturen. Insofern geht meine Kritik weiter als die von $S c b u-$ mann aufgezeigte empirische Fragwürdigkeit der von allen Sicherungstheorien unterstellten »Gefährlichkeits«-Annahmen. Mir geht es um die illiberale Tendenz aller täterspezifischen Theorien, die Schwere der Tat zu ignorieren und auf den „Täter" als unerwünschtes oder zu resozialisierendes Objekt staatlicher Prävention zu blicken.

Im Unterschied zu der von mir vertretenen grundsätzlichen Ablehnung des Sicherungsgedankens geht es Schumann darum, ihn als im Prinzip legitimen Grund für eine Freiheitsstrafe rational zu kontrollieren. Zu diesem Zweck zeigt er die Fehlerquellen auf, die jede Gefährlichkeitsprognose zwangsläufig hat, er verzichtet aber auf eine Theorie, die den Sicherungsaspekt als Strafgrund grundsätzlich angreift. Skep-

5 Historısch geht diese moralisierende und wohl deshalb im Kern illıberale Schuldlehre auf M. E. Mayer (1901) zuruck. Die in fruheren Zeiten beliebte und von kunftigen Rıchtern repetitorhaft gelernte Formel lautete: der Charakter belastet, das Motiv entlastet. Spatestens 1968-1975 hat diese Formel ihre fruhere Beliebtheit eingebußt, da man dıe Nahe zur charakterologischen, von der natıonalsozialıstischen Mınderwertigkeitsdoktrin rezipıerten Tatertypologie gesehen hatte. Sie wurde aber bisweilen wiederholt, sogar be1 ansonsten sehr vorsichtigen Autoren wie Engisch, vgl. dens., ZStW 66, 1965, S. 339 ff. Zur nationalsozialistischen Tatertypenlehre vgl. exemplarisch (zum »Morder “) Frommel, JZ 1980, S. 599 ff. 
tisch stimmen ihn nämlich alle juristischen Legitimationsformeln, heißen sie nun Abschreckung, Ausgleich zurechenbarer Tatschuld oder indirekte Generalprävention $^{6}$. Er unterstellt juristischen Zuschreibungsmodellen (trotz ihrer ganz unterschiedlichen theoretischen Anknüpfungen) eine Art »deformation professionelle«. Diese führe dazu, daß die gesamte Berufsgruppe (wo bleiben die Strafverteidiger?) dem Strafrecht grundsätzlich eine größere Wirkung zuschrieben, als tatsächlich berechtigt wäre. Als Sozialwissenschaftler wählt er daher - bewußt gegen diese normativen Debatten gewendet - einen streng empirischen Ausgangspunkt. Danach wäre eine Sicherungstheorie legitim, wenn ein Sicherungseffekt meßbar wäre. Wenn es also gelänge, eine Unterstellung, deren Realitätsgehalt er bezweifelt, eine Gruppe besonders gefährlicher potentieller Täter zu fixieren und sie ausschließlich wegen ihrer Gefährlichkeit, d.h. aus präventiven Gründen zu sichern, dann wäre dieses Straf(-und Maßregel)prinzip »utilitaristisch « (und damit scheinbar kühl zweckrational kalkulierend) begründbar. Ich fürchte, dies ist eine Verharmlosung des Problems und ein theoretischer Zugriff auf normative Probleme der Gesellschaft, die im Ansatz verfehlt ist, da empirische und normative Probleme eine unterschiedliche Struktur aufweisen, so daß auch Ideologiekritik jeweils beide Aspekte berücksichtigen sollte.

Ein erster, noch sehr normativistischer Einwand lautet: Freiheitsentzug zur Sicherung der Gesellschaft vor einzelnen Personen kann nur eine Angelegenheit des Unterbringungsrechts sein. Es handelt sich um eine (gesondert zu legitimierende) Maßnahme, die im Prinzip nichts mit Strafrecht zu tun hat und nur aus historischen Gründen - wegen des noch zulässigen, aber zunehmend seltener genutzten Maßnahmerechts (sog. Zweispurigkeit von Strafe und Maßnahme nach $\$ \$ 62 \mathrm{ff}$. StGB) - im StGB geregelt ist. Mit anderen Worten: legitim kann Strafe ohnhin nur sein, wenn sie nicht nur »polizeilich«, sondern auch in einem moralisch-normativen Sinne notwendig ist. Folgt man dieser, fast allen philosophischen, juristischen und auch den funktionalistischen (Durkheim als Beispiel) Theorien zugrunde liegenden Annahme, dann muß es gute Gründe geben für die Notwendigkeit einer strafrechtlichen Sanktion.

\section{Tat- und Täterorientierung}

Alle liberalen Theorien sehen diese guten Gründe in der die Strafgewalt begrenzenden Argumentation, der Normverstoß müsse so gravierend sein, daß das "ultima ratio «-Prinzip eine strafrechtliche Reaktion rechtfertige. Ultima ratio meint in diesem (zweifellos normativ und nicht empirisch gemeinten) Zusammenhang nicht »effektive, meßbare Wirkung «, sondern einsichtig begründbare Bewertung des Normverstoßes als fundamental. Dieser kritische Ansatz ist allen tatorientierten Modellen gemeinsam. Hingegen kennzeichnet täterorientierte Modelle ein moralisierender (Lebensführungsschuld) und/oder ein den Täter offen abwertender Ton (Gefährlichkeit, Minderwertigkeit). Auch das Etikett »sozialgefährlich« wertet $a b$, und zwar in einer viel radikaleren Weise als das sog. sozialethische Unwerturteil im eher behäbigen Schuldstrafrecht. Schon dies spricht in meinen Augen gegen eine Sicherungstheorie. Genauer: nicht nur der Sicherungsgedanke, sondern jede Täterorientierung verweist auf ein Denkschema, das prinzipiell und nicht nur gelegentlich dazu neigt, situative Rahmenbedingungen einer Handlung (sei sie wiederholt oder vereinzelt) zu ignorieren oder - was noch schlimmer ist - eine ungünstige Situation in eine negative Eigenschaft des Täters (deshalb sei er nämlich

6 Schumann, Positive Generalpravention, Ergebnisse und Chancen der Forschung, 1989, insb. S. 52 f. 
gefährlich) umzudeuten. Im angelsächsischen Kontext waren und sind es sozialdarwinistische, in Deutschland offen autoritäre bis nationalsozialistische Denkansätze, die so (ver)urteilen und die sich alle am Sicherungsgedanken orientieren bzw. orientiert haben.

\section{Historischer Rückblick}

$\mathrm{Zu}$ erinnern ist an die strafrechtliche und polizeiliche "Bekämpfung " der sog. Gewohnheitsverbrecher nach dem »Gesetz gegen gefährliche Gewohnheitsverbrecher « vom 24. 1 I. 1933. Es gab der Strafjustiz sehr weitgehende Kompetenzen gegen sog. "Hang «-Täter bzw. Jugendliche mit "schädlichen Neigungen«. Das Gewohnheitsverbrechergesetz gehörte zu den ersten Maßnahmen der Nationalsozialisten, die Kontinuität zu früheren Entwürfen und die Diskontinuität zwischen liberaler "Reform « und »völkischer Erneuerung « des Rechts zu demonstrieren. Sie waren dabei so erfolgreich, daß sowohl 1933 als auch 1945 dieses Gesetz, das viele sog. "Gewohnheitsverbrecher ins Konzentrationslager gebracht hatte, als nicht typisch nationalsozialistisch eingestuft wurde. Im Gegenteil, die breite Akzeptanz nach 1933 führte zu einer extensiven Nutzung: In den Jahren 1934 bis 1943 wurden etwa 16000 Personen offiziell zur Sicherheitsverwahrung verurteilt. Vollstreckt wurde diese in Konzentrationslagern. Bis heute sind die in Lagern ermordeten "Kriminellen « keine anerkannten Opfer des Nationalsozialismus. Nach 1945 (also bis heute) blieb der zweifelhafte Begriff des »Hangs « ( $\$ 66 \mathrm{StGB}$ ) ebenso wie die »schädliche Neigung « $(\$ 17$ Abs. 2 JGG) beibehalten. In der Praxis seit der Neufassung des $\$ 66$ StGB im Jahr 1970 spielt allerdings zumindest die Sicherungsverwahrung nur noch eine marginale Rolle und sollte ersatzlos entfallen?

\section{Sicherung als eigenständiger Strafzweck ist überflüssig und illegitim}

Die Unklarheit, wer denn nun »sozialgefährlich « sei und wer nicht, führt Schumann zu einer sozialwissenschaftlichen Kritik an der empirischen Validität von Prognoseverfahren. Normative Zweifel an der Berechtigung des Sicherungsgedankens deutet er an, aber in einer Weise, die irritiert. Betroffen seien Personen, die für "Taten, die sie noch nicht begangen « haben, bestraft werden sollten, ist in der Einführung zu lesen. Ich gestehe Schumann zu, daß diese Qualifikation sich für eine kurze polemische Bemerkung eignen könnte, aber offenbar meint er mehr, nämlich daß die Antizipation künftiger Taten eine prinzipiell zulässige Begründung sei, wenn es nur Verfahren der validen Prognose gäbe. In dieselbe Richtung weist auch die Bemerkung: "Auch Abolitionisten akzeptieren Haft zur Krisenintervention. Uneinig ist man über die Größe der Gruppe, die Sozialgefährlichkeit der erwartbaren Taten und eine Identifikation dieser >Gefährlichen` durch Prognoseverfahren«. Abgesehen davon, daß ich keinen Abolitionisten (außer Schumann und den von ihm zitierten Bianchi) kenne, der eine Sicherungshaft positiv legitimieren würde, wenn sie nur offen zugegeben würde, so kann doch dieser Hinweis nur bedeuten, daß Sicherung

7 Vgl. zum Kontext: Frommel, Praventionsmodelle in der deutschen Strafzweck-Diskussion. Beziehungen zwischen Rechtsphilosophie, Dogmatik, Rechtspolitik und Erfahrungswissenschaften, 1987; zum Gewohnheitsverbrechergesetz: dies., Von der Strafrechtsreform zur "Rechtserneuerung, in: Rottleuthner, $\mathrm{Hg}$., Recht, Rechtsphilosophie und Nationalsozialismus, Beiheft 18 des ARSP, 1983 , S. 45 ; dies., Verbrechensbekämpfung im Nationalsozialismus, in: Festschrift für Sten Gagnér, hg. von Michael Stolleis u.a., 1991, S. 47.

Zu Einzelfragen wie dem Begriff des „Hangs«: dies., Die Anordnung der Sicherungsverwahrung bei Gelegenheitstaten, NJW 1981, S. 1083 ; ferner zur Täterorientierung: dies., Die Bedeutung der Tätertypenlehre bei der Entstehung des $\$ 211$ StGB im Jahre 1941, JZ 1980, S. 559 
der einzige rational legitimierbare utilitaristische Strafgrund wäre. Zu dieser Interpretation paßt die Forderung am Ende des Textes: "zumindest muß, wenn Haft für noch nicht begangene Straftaten verbüßt werden soll, die Annahme zukünftiger Gefahr auf triftige und möglicherweise auch vorwerfbare Kriterien gestützt werden «.

Irritierend sind diese Bemerkungen schon deswegen, weil sie den Etikettierungsansatz, d.h. die kritische Analyse der jeweiligen informellen und formellen Regeln, Handlungen als »kriminell « zu definieren, umdeuten in ein rechtspolitisches Programm einer "rationalen « Prävention. Letztere wird gesehen als der in meinen Augen fiktive Versuch, empirisch greifbare Kriterien zum »Schutz der Allgemeinheit vor rückfallträchtigen Tätern « zu formulieren. Abolitionisten sind sich keinesfalls einig, daß es in einem Staatswesen konsensfähige Kriterien für "Sozialgefährlichkeit « geben könnte. Einfacher hat es da die Theorie der indirekten Generalprävention. Es lassen sich nämlich konsensfähig fundamentale Normen auflisten (das Verbot von Brachialgewalt etwa), deren Verletzung »eigentlich « jede Gesellschaft ahnden müßte, aber diese Normen bezichen sich auf Taten, sie sind also prinzipiell tat- und nicht täterorientiert. Täterorientierte Ansätze sind hingegen per se fragwürdig, da sie mit Annahmen der angeborenen oder erworbenen Minderwertigkeit operieren müssen. Interpretiert man Schumanns Text als Plädoyer für eine utilitaristische Straftheorie und ein täterorientiertes Strafrecht, dann zeigt sich eine fast tragische Naivität, eine unhistorische Sicht, die schon vor hundert Jahren die Bewegung der »défense sociale k kennzeichnete. Unhistorisch ist sie u.a. deshalb, weil sie die historisch mächtigen präventiven Strafzwecke eines Reformers wie etwa Franz Liszt als "liberal« einstuft. Dieselbe Sicht führte nach 1945 dazu, auch den überkommenen Sicherungsgedanken als legitimen Strafzweck anzusehen. Mittlerweile erkennen viele, daß nur ein konsequent tatorientiertes Strafrecht rechtsstaatlich und liberal ist. Die Zurechnung als "Schuld « ist eine legitime Konsequenz des Verantwortungsprinzips. Es darf nur zu strafrechtlichen Sanktionen führen, wenn die Lebensbedingungen des Beschuldigten die Zuschreibung von Verantwortung auch zulassen. So gesehen sind heute täterorientierte utilitaristische Straftheorien fragwürdiger denn je. Folgt man diesem Ansatz, ergibt sich ein Vierfelder-Schema, das zwischen tatorientierten und täterorientierten Zurechnungs- und Präventionsmodellen unterscheidet. Eine liberale Straftheorie erfordert eine Theorie der Begrenzung der Strafgewalt. Die Zurechnung einer Tat impliziert eine moralische Dimension der Verantwortlichkeit. Das Sanktionensystem verweist zwar darüber hinaus auch auf zweckrationale Konzepte der klugen Wahl geeigneter Reaktionsformen. Strafbegrenzend sind auch tatorientierte Argumente der Zurechnung. Eher illiberal sind hingegen moralisierende, d.h. täterorientierte Unwerturteile und täterorientierte Konzepte der Verbrechensbekämpfung. Sie transformieren das Verantwortungs- und Präventionsprinzip zu einem ethisierenden Vergeltungsrecht und/oder einem flächendeckenden Präventions- oder Bekämpfungsrecht und führen insbesondere zur Ausgrenzung der Personen, die mehrfach mit einem Unwerturteil belegt worden sind. Moralische Diffamierung (böse oder minderwertig) und scheinbar zweckrational motivierte Zuschreibungen (wie abweichend, unerwünscht und gefährlich) sind dabei in der Realität austauschbar. Daher gibt es gute Argumente, Ethisierung und Instrumentalisierung als die Formen liberalen Denkens zu fixieren. Sie haben beide eine lange Tradition. Karl Binding etwa und das klassische generalpräventive Vergeltungsstrafrecht sind geradezu paradigmatisch für eine Kombination von Minderwertigkeits- und Gefährlichkeitsargumenten: 
„Unbedingt lehne ich ab die Herabwürdigung der Strafe zur polizeilichen Sicherungsmaßregel. Sie ist etwas Anderes, Höheres, Edleres!« Aber man konne die Strafe schärfen für »verbrecherische Hartnackigkeit«, um die »Sippschaft« unschädlich zu machen. Man konne die Rückfallstrafe steigern »eventuel] bis zur Todes- oder Freiheitsstrafe auf Lebenszeit « ${ }^{8}$

Gegen Liszts Sicherungsstrafe sprach also in den Augen Bindings nicht deren Härte, die mit dem Schuldprinzip nicht in Einklang stehe, sondern nur die begriffliche Herabwürdigung der Strafe zum Sicherungsmittel. Dies führt uns zu Franz v. Liszts ambivalenter Theorie. Sie impliziert eine tatorientierte Zuschreibung und eine täterorientierte Prävention, ist also nach dem hier vorgeschlagenen Schema »illiberal«, was bestätigt wird durch die Tatsache, daß es keine begrifflichen Argumente gegen eine Zuschreibung nach Gesichtspunkten der Gefährlichkeit gibt. ${ }^{9}$ Aber sie postuliert immerhin noch eine im Ansatz zweckrationale Strafzumessung, vermeidet also die Wucht der moralischen Degradierung. Während des Dritten Reiches gab es daher nicht ohne Grund die bemerkenswerte Sprachregelung, die Lisztsche Tätertypologie zu loben, aber seine "rationalistische Verbrechensauffassung scharf zurückzuweisen. Besonders prägnant findet sie sich bei Edmund Mezger:

"Die soziologische Verbrechensauffassung ist daher in ihrer letzten Konsequenz eines okonomischen Materialismus das Ende der Verantwortlichkeit des einzelnen und der Selbständigkeit des geistigen Lebens überhaupt, auch das Ende aller Kultur. Das Schöpferische im Menschen erstickt im Untermenschentum ". ${ }^{10}$

I 944 schreibt derselbe Autor über die Rassengesetzgebung des nationalsozialistischen Staates:

"Der konstitutions- und erbbiologischen Auffassung kommt dabei besondere Bedeutung zu, weil beide die Möglichkeit ergeben, die einzelnen Züge und Seiten der Persönlichkeit nicht nur für sich, sondern in dem größeren Zusammenhang der wesensmäßigen und blutmäßigen Bedingtheiten zu sehen. Der Gedanke der Ausmerzung volks- und rasseschadlicher Teile der Bevolkerung findet hier seinen Rückhalt «."

Fragwürdig ist der Sicherungsgedanke also nicht nur in dem von Schumann kritisierten Sinn, weil er für Taten, die noch nicht begangen worden sind, »bestraft ", sondern weil er zwangsläufig »minderwertige« (krank oder böse) und »normale« bzw. »wertvolle« Menschen konstruiert, um selektiv sein zu können.

8 K. Bindıng, Grundriß des Strafrechts AT, 1906 (Vorwort).

9 Vgl. nur M. Grunhut, Gefahrlichkeit als Schuldmoment, Festgabe Aschaffenburg, 1926, S. 8 ff.

10 E. Mezger, Kriminalpolitık und ihre kriminologischen Grundlagen, 1934, S. I 72

II Ders., 3. Aufl. 1944, S. 247. 